UL-NTZ 22/98, KANAZAWA-98-13, ITEP-TH-44/98

September 5, 1998

\title{
Embedded Topological Defects in Electroweak Theory: From Percolating Networks to Sphalerons*
}

\author{
M. N. Chernodub ${ }^{\mathrm{a}}$, F. V. Gubarev ${ }^{\mathrm{a}}$, E.-M. Ilgenfritz ${ }^{\mathrm{b}}$ and A. Schiller ${ }^{\mathrm{c}}$ \\ aTEP, B. Cheremushkinskaya 25, Moscow 117259, Russia \\ bInstitute for Theoretical Physics, Kanazawa University, Kanazawa 920-1192, Japan \\ 'Institut für Theoretische Physik and NTZ, University of Leipzig, D-04109 Leipzig, Germany
}

\begin{abstract}
New defects ( $Z$-vortices and Nambu monopoles) are found to become thermodynamically relevant for the broken phase near to the (weakly first order) electroweak phase transition, and below the crossover for higher Higgs mass. The symmetric phase is characterized by vortex condensation (percolation). The percolation transition persists in the crossover region. The quasiclassical nature of the vortices is supported by correlations with Higgs field and gauge field energy. Sphalerons are shown to be related to monopolium bound states.
\end{abstract}

\section{INTRODUCTION}

Is the electroweak phase transition accompanied by the condensation of some defects? What is happening with the defects at the crossover region? Is there a range of parameters supporting a dilute gas of such defects in the broken phase and how are they related to the well-known sphaleron configurations? These questions have been explored during the last year within the $S U(2)$ Higgs model.

The $Z$-vortex string 1.2 corresponds to the Abrikosov-Nielsen-Olesen [3] vortex solution related to the Abelian subgroup of $S U(2)$ embedded into the electroweak theory [4, [5]. Zstrings have been demonstrated to be unstable both at zero and at finite temperatures [6]. Sphalerons [1.7, 8] are unstable configurations as well, being transition states (saddle points) between vacua with different Chern-Simons number. Classical sphalerons have been constructed on the lattice in Refs. [9] by a specific saddle point cooling technique (these configurations have been important for our project). Baryon asymmetry should not be washed out by sphaleron processes after the electroweak symmetry breaking has been completed. This requirement puts strin-

\footnotetext{
*Combined contribution covering the talks at LAT'98 by
} M. N. Chernodub and E.-M. Ilgenfritz gent conditions on the strength of the phase transition because the activation energy of these barriers strongly depends on the strength of the electroweak phase transition [10].

Recently, lattice studies have shown (see 11 for a conclusive summary) that neither the simplified $S U(2)$ Higgs model nor the $S U(2) \times U(1)$ model have a thermal transition of the necessary strength, given the experimental bounds on the Higgs mass. This observation has triggered our interest to investigate whether, for Higgs masses below the end of the phase transition [12] and in the crossover region above, topological excitations might be excited with a non-negligible statistical weight. These historical circumstances might explain why defects in the context of the electroweak phase transition are a rather new topic within lattice gauge theory.

In this contribution we briefly describe our lattice definitions and present selected results, some of them having not been published before. Details can be found in Refs. 13 15. Our study has been performed within the dimensionally reduced $S U(2)$ Higgs theory which provides an effective representation of the electroweak theory in some range of Higgs masses and at temperatures near to the electroweak scale. For the formulation of the lattice version of the effective theory see the talk of A. Schiller at this conference [16]. 
The lattice gauge coupling $\beta_{G}=4 /\left(a g_{3}^{2}\right)$ (with $\left.g_{3}^{2} \approx g_{4}^{2} T\right)$ gives the lattice spacing in units of temperature, and the hopping parameter $\beta_{H}$ substitutes $m_{3}^{2}$ driving the transition. The parameter of the phase transition or the crossover can be translated into temperature $T_{c}$ and a Higgs mass $M_{H} \approx M_{H}^{*}$ (see e.g. Ref. [17] and references therein). Notice that the Higgs field is usually written as a multiple of a $S U(2)$ matrix $\Phi$, here we need it in the form of a 2-component isospinor $\phi$.

\section{DEFINITIONS}

The gauge invariant and quantized lattice definition 13. of elementary $Z$-vortices and Nambu monopoles is closely related to the definition in the continuum theory [2]. First we define a composite adjoint unit vector field

$n_{x}=n_{x}^{a} \sigma^{a}, \quad n_{x}^{a}=-\frac{\phi_{x}^{+} \sigma^{a} \phi_{x}}{\phi_{x}^{+} \phi_{x}}$,

$\sigma^{a}$ are Pauli matrices. For the following purpose, $n_{x}$ plays a role similar to the adjoint Higgs field in the definition of the 't Hooft-Polyakov monopole [18] within the Georgi-Glashow model. It helps to define the gauge invariant flux $\bar{\theta}_{p}$ through the plaquette $p=\{x, \mu \nu\}$,

$\bar{\theta}_{p}=\arg \left(\operatorname{Tr}\left[\left(1+n_{x}\right) V_{x, \mu} V_{x+\hat{\mu}, \nu} V_{x+\hat{\nu}, \mu}^{+} V_{x, \nu}^{+}\right]\right)$

via the projected links

$V_{x, \mu}(U, n)=U_{x, \mu}+n_{x} U_{x, \mu} n_{x+\hat{\mu}}$.

The auxiliary Higgs field $n_{x}$ is covariantly constant with respect to these $S U(2)$-valued links. Originally, Nambu monopoles are topological defects of an Abelian field which is defined in the unitary gauge $\left(\phi_{x}=(0, \varphi)^{T}\right.$ and $\left.n_{x}^{a} \equiv \delta^{a 3}\right)$ by the phases $\theta_{l}^{u}=\arg U_{l}^{11}$. This field behaves as a compact Abelian field with respect to residual Abelian gauge transformations which leave the unitary gauge condition intact. The gauge invariant flux would be extracted from its plaquettes $\theta_{p}^{u}$ as usual: $\bar{\theta}_{p}=\left(\theta_{p}^{u}-2 \pi m_{p}\right) \in[-\pi, \pi)$. The magnetic charge of Nambu monopole cubes and the $Z$-vorticity number of plaquettes can alternatively be defined in a gauge invariant way 13] referring to (2). The monopole charge $j_{c}$ inside the cube $c$ is defined in terms of the gauge invariant fluxes (2) penetrating the surface $\partial c$ :

$j_{c}=-\frac{1}{2 \pi} \sum_{p \in \partial c} \bar{\theta}_{p}$.

The $Z$-vorticity number of the plaquette $p$ is defined 13] as follows:

$\sigma_{p}=\frac{1}{2 \pi}\left(\chi_{p}-\bar{\theta}_{p}\right)$,

where $\bar{\theta}_{p}$ has been given in (2), and

$\chi_{p}=\chi_{x, \mu \nu}=\chi_{x, \mu}+\chi_{x+\hat{\mu}, \nu}-\chi_{x+\hat{\nu}, \mu}-\chi_{x, \nu}$,

is a plaquette variable written in terms of the Abelian links $\chi_{x, \mu}=\arg \left(\phi_{x}^{+} V_{x, \mu} \phi_{x+\hat{\mu}}\right)$.

A $Z$-vortex is formed by links $l=\{x, \rho\}$ of the dual lattice which are dual to plaquettes $p=\{x, \mu \nu\}$ which carry a non-zero vortex number (5): ${ }^{*} \sigma_{x, \rho}=\varepsilon_{\rho \mu \nu} \sigma_{x, \mu \nu} / 2 . \quad Z$-vortex trajectories are either closed or begin/end on Nambu (anti-) monopoles:

$\sum_{\mu=1}^{3}\left({ }^{*} \sigma_{x-\hat{\mu}, \mu}-{ }^{*} \sigma_{x, \mu}\right)={ }^{*} j_{x}$.

This is how we actually localize Nambu monopoles.

When the embedded defects are physical excitations with some intrinsic size, one should be able to describe them on finer lattices as extended topological objects. An extended monopole (vortex) of physical size $k a$ is defined on $k^{3}$ cubes ( $k^{2}$ plaquettes, respectively) 19,21. The charge of monopoles $j_{c(k)}$ on bigger $k^{3}$ cubes $c(k)$ is constructed analogously to that of the elementary monopole (1) with elementary $1 \times 1$ plaquettes in terms of $V_{x, \mu}$ replaced by $n \times n$ Wilson loops (extended plaquettes). In pure gauge theory, within the maximally Abelian gauge, these objects are called type-I extended Abelian monopoles We have numerically investigated the behavior of the type-I extended vortices of some fixed physical size on a series of finer and finer lattices towards the continuum limit. Our results suggest that the continuum limit for type-I extended vortices may exist. We remark that an alternative, typeII, construction [19] of extended objects fails to 
show a physically sensible behavior towards continuum limits.

\section{RESULTS}

We have scanned the phase transition with elementary defects at $M_{H}^{*}=30 \mathrm{GeV}$ (strong first order) and $70 \mathrm{GeV}$ (weak first order) for $\beta_{G}=12$ on a relatively small lattice $16^{3}$. At the lower Higgs mass we observed a discontinuity of the densities $\rho_{m}=\sum_{c}\left|j_{c}\right| / L^{3}$ of Nambu monopoles and $\rho_{v}=\sum_{p}\left|\sigma_{p}\right| /\left(3 L^{3}\right)$ of vortices. Simultaneously we have measured the percolation probability $C=\lim _{r \rightarrow \infty} C(r)$ derived from the cluster correlation function [20]

$C(r)=\frac{\sum_{x, y, i} \delta_{x \in{ }^{*} \sigma^{(i)}} \delta_{y \in{ }^{*} \sigma^{(i)}} \cdot \delta(|x-y|-r)}{\sum_{x, y} \delta(|x-y|-r)}$,

where only those connected clusters ${ }^{*} \sigma^{(i)}$ of vortex lines ( $i$ labels distinct vortex clusters) contribute to the nominator which pass through the points $x$ and $y$. Connected clusters ${ }^{*} \sigma^{(i)}$ are called percolating clusters if they contribute to the limit $C$.
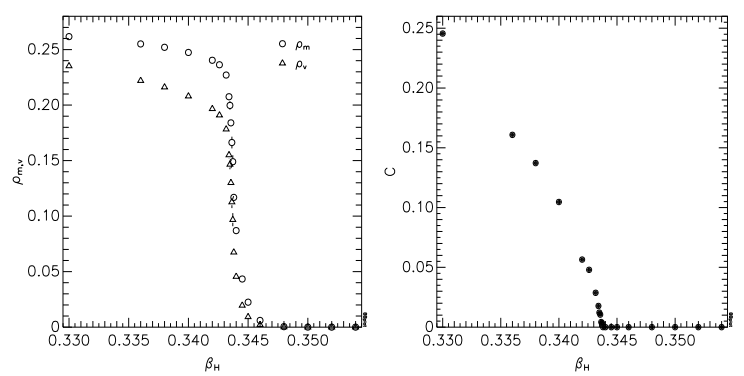

Figure 1. Densities of elementary Nambu monopoles $\rho_{m}$ and of $Z$-vortices $\rho_{v}$ (left) for Higgs mass $M_{H}^{*}=$ $70 \mathrm{GeV}$ at gauge coupling $\beta_{G}=12$; Percolation probability $C$ of $Z$-vortex trajectories (right).

The percolation probability has a finite jump to zero at the pseudocritical $\beta_{H c}=0.3411$ for $M_{H}^{*}=30 \mathrm{GeV}$. The same study at $M_{H}^{*}=70$
$\mathrm{GeV}$, already near to the endpoint of the first order transition, is summarized in Figure 1 and shows the percolation probability continuously vanishing towards $\beta_{H c}=0.34355$. This is an inflection point of the densities $\rho_{m}$ and $\rho_{v}$ where they are approximately equal to half of their values in the symmetric phase. For $\beta_{H}>\beta_{H c}$ the densities decrease exponentially.

For the case $M_{H}^{*}=30 \mathrm{GeV}$ (where we find zero densities of elementary defects on the broken side) we have made some tests whether the densities of extended defects on the symmetric side admit a continuum limit. We have chosen elementary vortices and monopoles on a lattice simulated at $\beta_{G}^{(1)}=8$ and compared them with extended defects of size $k=2,3$ for simulations at $\beta_{G}^{(2)}=16$ and $\beta_{G}^{(3)}=24$, respectively. A lattice size $16^{3}$ corresponding to a shrinking volume has been taken for all three cases because we did not expect severe finite size effects at such a strong transition.
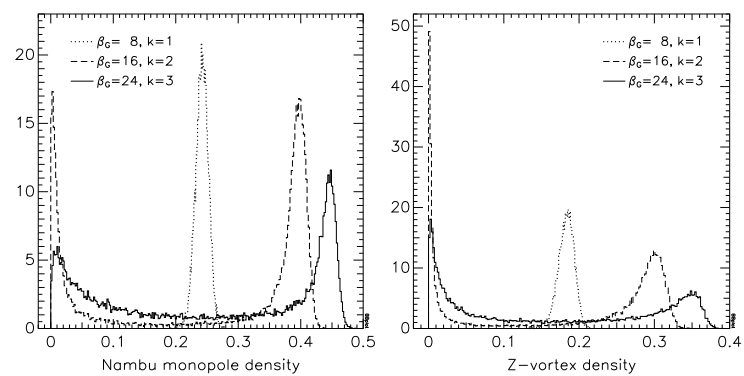

Figure 2. Density distributions of Nambu monopoles (left) and $Z$-vortices (right) of fixed thickness at pseudocriticality for different gauge couplings for Higgs mass $M_{H}^{*}=30 \mathrm{GeV}$.

This (preliminary) test is shown in Figure 2 in the form of histograms of average vortex and monopole densities per configuration. The measurements have been performed near to the respective $\beta_{H c}$ for the three values of $\beta_{G}$ and gave always a two-state signal with a density distribution peaking at or near to zero on the symmetric side. There is no way to perform an additive renormalization of the density at the broken side 
by taking differences between the phases (in analogy to the Higgs modulus squared $\rho^{2}$, Ref. [21]). With a box size $a\left(\beta_{G}=8\right)(a \simeq 1 / T)$ we seem to be at the upper edge of the size distribution of physical vortices. With increasing $\beta_{G}$, our construction for extended defects seems to describe the vorticity better. This might explain the monotonous increase to some (still unknown) continuum density of vortices/monopoles.
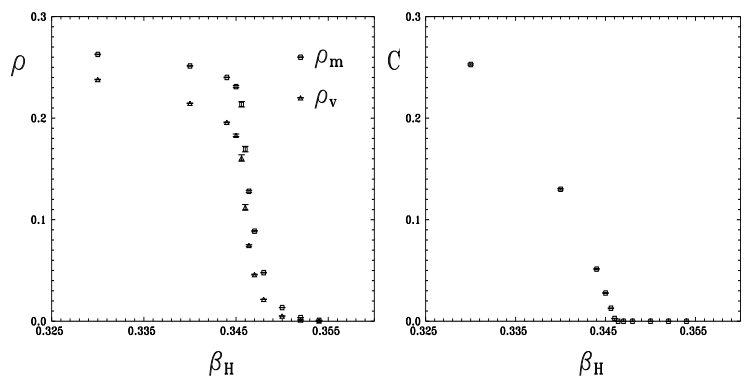

Figure 3. Densities of elementary Nambu monopoles $\rho_{m}$ and of $Z$-vortices $\rho_{v}$ (left) for Higgs mass $M_{H}^{*}=$ $100 \mathrm{GeV}$ at gauge coupling $\beta_{G}=12$; Percolation probability $C$ of $Z$-vortex trajectories (right).
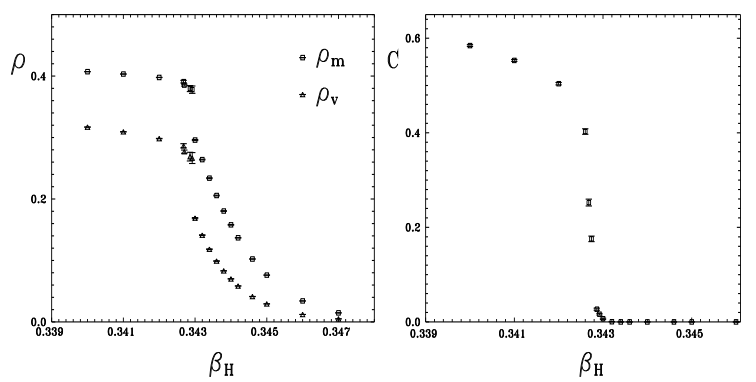

Figure 4. Same as Figure 3 for extended defects of size $k=2$.

Physically the crossover region is more interesting since we can study the properties of isolated defects in a not-so-dilute environment. We show in Figure 3 the behavior of densities and percolation probability $C$ vs. $\beta_{H}$ for $M_{H}^{*}=100$ $\mathrm{GeV}$ at $\beta_{G}=12$ for elementary defects. We were wondering whether there is an universal percolation temperature for vortices of extendedness $k a$ at respective $\beta_{G}^{(k)}=k \beta_{G}^{(1)}$. In order to prevent bigger finite size effects, now we kept the physical volume constant using $(k L)^{3}$ lattices. The percolation probability $C$ and the densities are shown in Figure 4 vs. $\beta_{H}$ as measured for $k=2$ on a $32^{3}$ lattice. From all critical $\beta_{H c}^{(k)}$ values we estimate $T^{\text {perc }}=170$ or $130 \mathrm{GeV}$ (without or with $t$-quarks). This corresponds to a Higgs mass $M_{H}=94$ or $103 \mathrm{GeV}\left(c f . M_{H}^{*}=100 \mathrm{GeV}\right)$.
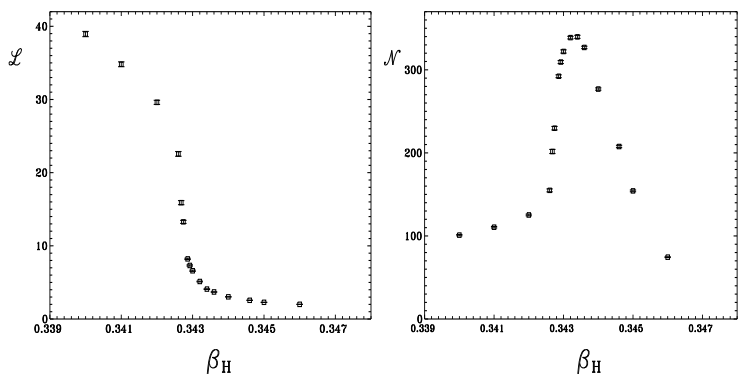

Figure 5. Average length $\mathcal{L}$ per $Z$-vortex cluster (left) and average number $\mathcal{N}$ of mutually disconnected $Z$-vortex clusters per lattice configuration $k=2$ objects on a $32^{3}$ lattice at $\beta_{G}=16$.

The cluster structure of the configurations changes drastically at this temperature. We illustrate this for $k=2$ vortices in Figure 5 with the average number of clusters and the average length per cluster vs. $\beta_{H}$.

Our results obtained in an effective $3 D$ equilibrium approach suggest a scenario with a (few) percolating clusters decaying into smaller ones, vortex rings and monopoliums, a process to be described by a kinetic approach to the crossover. Note that the average length per cluster has a long tail at a level of two. We propose to interpret the half density $\rho_{v} / 2$ as the density of spalerons on the broken side of the crossover. We support this conjecture by what we have found [13] to be the signature of a classical lattice sphaleron with respect to the new ( $Z$-vortex and Nambu monopole) degrees of freedom. Figure 6 shows one of the solutions of Ref. [9] with a Nambu 
monopole-antimonopole pair in the center, compared with a view in the maximally Abelian gauge.
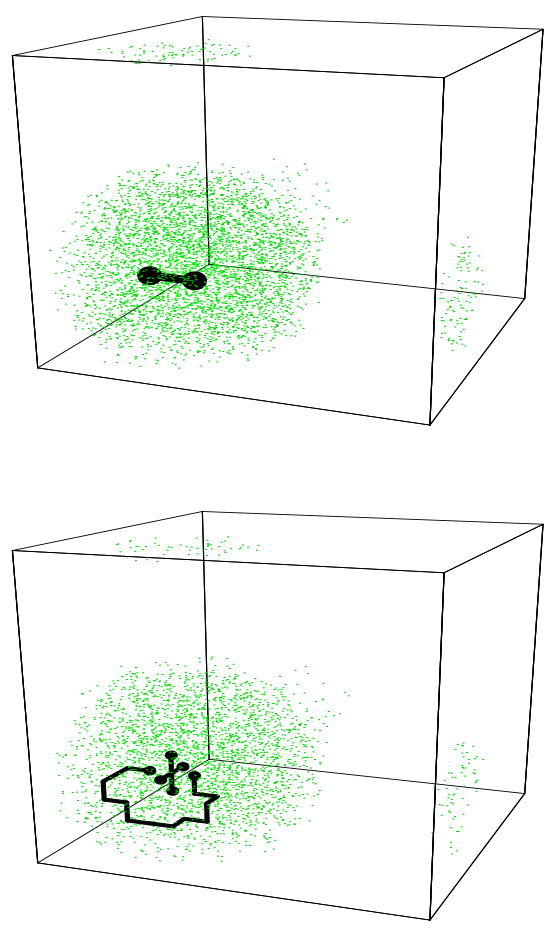

Figure 6. Classical lattice sphaleron with a Nambu monopole-anti-monopole pair bound by a $Z$-vortex string in the center (above) and with Abelian monopoles and Abelian vortices in the maximally Abelian gauge (below). The density of the clouds is proportional to the decrease in Higgs field modulus.

One might argue that the defects counted by the lattice operators (4) and (5) might be mere artifacts having nothing in common with the vortex solutions in the continuum. Figure 7 shows that this is not the case. Here the average gauge field action per plaquette and the Higgs field modulus squared averaged over the corners of a plaquette is shown as function of $\beta_{H}$, separately for plaquettes with $\sigma_{p} \neq 0$ and $\sigma_{p}=0$. The data refer to
$M_{H}=100 \mathrm{GeV}$ and $\beta_{G}=8$.

One can clearly see that the modulus of the Higgs field is smaller near to the vortex trajectory than outside the vortex for all values of the coupling $\beta_{H}$. Moreover, the value of the gauge field energy near to the vortex center is larger than in the bulk (the increasing error of the gauge field action inside the vortex at the largest $\beta_{H}$ reflects the small number of plaquettes with nonvanishing vorticity).
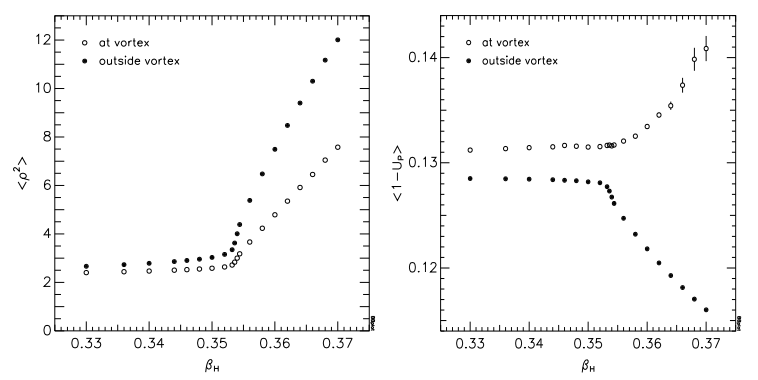

Figure 7. Average squared Higgs modulus (left) inside and outside an elementary $(k=1) Z$-vortex on both sides of the percolation transition. Right: the same for gauge field energy.

These results show that $Z$-vortices are physical objects which resemble the characteristic features of the classical vortex solutions in continuum: the vortices carry the excess of the gauge field energy and the Higgs modulus decreases near to the vortex center.

\section{OUTLOOK}

In future we have to complete these studies by a more systematic exploration of the continuum behavior/size distribution of defects. Furthermore we plan to extend our considerations to more realistic models with non-zero Weinberg angle. Last but not least, Euclidean simulations seem to be necessary to clarify the connection of the dynamics of vortices with the evolution of the ChernSimons number. This may shed some light on the baryogenesis scenario [22 based on the dynamical properties of the $Z$-vortex string network. 
ACKNOWLEDGMENTS

The authors have benefited from discussions with L. McLerran, M. Müller-Preussker, M. I. Polikarpov, V. A. Rubakov, K. Rummukainen, K. Selivanov, T. Suzuki and P. G. Tinyakov. We are very grateful to Pierre van Baal and Margarita Garcia-Perez for providing the sphaleron lattice configurations.

M. N. Ch. and F. V. G. were partially supported by the grants INTAS-96-370, INTASRFBR-95-0681, RFBR-96-02-17230a and RFBR96-15-96740.

\section{REFERENCES}

1. N. S. Manton, Phys. Rev. D28 (1983) 2019.

2. Y. Nambu, Nucl. Phys. B130 (1977) 505.

3. A. A. Abrikosov, Sov. Phys. JETP 32 (1957) 1442; H. B. Nielsen and P. Olesen, Nucl. Phys. B61 (1973) 45.

4. T. Vachaspati and M. Barriola, Phys. Rev. Lett. 69 (1992) 1867.

5. M. Barriola, T. Vachaspati and M. Bucher, Phys. Rev. D50 (1994) 2819.

6. R. Holman, S. Hsu, T. Vachaspati and R. Watkins, Phys. Rev. D46 (1992) 5352.

7. R. F. Dashen, B. Hasslacher and A. Neveu, Phys. Rev. D10 (1974) 4138.

8. F. R. Klinkhamer and N. S. Manton, Phys. Rev. D30 (1984) 2212.

9. M. Garcia Perez and P. van Baal, Nucl. Phys. B429 (1994) 451; ibid. B468 (1996) 277.

10. V. A. Rubakov and M. E. Shaposhnikov, Usp. Fiz. Nauk 166 (1996) 493 (Phys. Usp. 39 (1996) 461).

11. M. Laine, these Proceedings.

12. M. Gürtler, E.-M. Ilgenfritz and A. Schiller, Phys. Rev. D56 (1997) 3888.

13. M. N. Chernodub, F. V. Gubarev and E.M. Ilgenfritz, Phys. Lett. B424 (1998) 106.

14. M. N. Chernodub, F. V. Gubarev, E.-M. Ilgenfritz and A. Schiller, e-Print Archive: heplat/9805016, Phys. Lett. B (in print).

15. M. N. Chernodub, F. V. Gubarev, E.-M. Ilgenfritz and A. Schiller, e-Print Archive: heplat/9807016, subm. to Phys. Lett. B.

16. E.-M. Ilgenfritz, A. Schiller and C. Strecha, these Proceedings, e-Print Archive: heplat/9808023.

17. M. Gürtler, E.-M. Ilgenfritz, J. Kripfganz, H. Perlt and A. Schiller, Nucl. Phys. B483 (1997) 383.

18. G. 't Hooft, Nucl. Phys. B79 (1974) 276; A. M. Polyakov, JETP Lett. 20 (1974) 194.

19. T. L. Ivanenko, A. V. Pochinskii and M. I. Polikarpov, Phys. Lett. B252 (1990) 631.

20. A. V. Pochinsky, M. I. Polikarpov and B. N. Yurchenko, Phys. Lett. A154 (1991) 194; T. L. Ivanenko, A. V. Pochinskii and M. I. Polikarpov, Phys. Lett. B302 (1993) 458; A. Hulsebos, Nucl. Phys. B (Proc. Suppl.) 34 (1994) 695.

21. K. Kajantie, M. Karjalainen, M. Laine, J. Peisa and A. Rajantie, Phys. Lett. B428 (1998) 334.

22. T. Vachaspati and G. B. Field, Phys. Rev. Lett. 73 (1994) 373; ibid. 74 (1995) (Errata); T. Vachaspati, in the Proceedings of the NATO Workshop on "Electroweak Physics and the Early Universe", Sintra, Portugal (1994); Series B: Physics Vol. 338, Plenum Press, New York (1994), e-Print Archive: hep-ph/9405286. 